\title{
Reactive oxygen species (ROS) accumulation induced by mononaphthalimide-spermidine leads to intrinsic and AIF-mediated apoptosis in HeLa cells
}

\author{
LIANHE YANG ${ }^{1,2}$, JIN ZHAO $^{1}$, YANQIN ZHU ${ }^{2}$, ZHIYONG TIAN ${ }^{3}$ and CHAOJIE WANG ${ }^{3}$ \\ ${ }^{1}$ Key Laboratory of Special Function Material, Henan University, Jinming Road, Kaifeng 475004; ${ }^{2}$ Basic Medical College, \\ Henan University of Traditional Chinese Medicine, Jinshui Road, Zhengzhou 450008; ${ }^{3}$ Key Laboratory of \\ Natural Medicine and Immune Engineering, Henan University, Jinming Road, Kaifeng 475004, P.R. China
}

Received November 3, 2010; Accepted January 3, 2011

DOI: $10.3892 /$ or.2011.1173

\begin{abstract}
Developing polyamine conjugates having the potential of transporting naphthalimide selectively into tumor cells is attractive. However, the evaluation of their cytotoxic mechanism has not been comprehensive. This study focused on the effects of mononaphthalimide spermidine (MNISpd) conjugate on apoptosis induction and the relationship between MNISpd-induced apoptosis and reactive oxygen species (ROS) in HeLa cells. Our findings indicated that $9 \mu \mathrm{M}$ MNISpd induced apoptosis in HeLa cells during a 48-h period. MNISpd induced apoptosis in HeLa cells following cytochrome c release, elevation of caspase 3/9 activity, apoptosisinducing factor (AIF) translocation and up-/down-regulation of $\mathrm{Bax} / \mathrm{Bcl}-2$ protein expression, respectively, and these effects were completely antagonized by pre-incubation with $10 \mathrm{mM}$ NAC for $2 \mathrm{~h}$. MNISpd induced significant ROS accumulation following up-regulation of polyamine oxidase (PAO) activity and complex variations in glutathione levels. It is concluded that MNISpd-induced apoptosis is related to intrinsic caspase-dependent and AIF-mediated caspaseindependent apoptosis pathways in HeLa cells. MNISpdinduced apoptosis correlates to MNISpd-induced ROS production resulting from GSH (reduced form of glutathione) pool depletion, and PAO is likely to be the source of ROS.
\end{abstract}

\section{Introduction}

Naphthalimides and their related derivatives have been extensively evaluated as DNA intercalating anti-tumor compounds (1-4). Many analogues of mononaphthalimide have been

Correspondence to: Professor Jin Zhao, Key Laboratory of Special Function Material, Henan University, Jinming Road, Kaifeng, 475004; or Professor Chaojie Wang, Key Laboratory of Natural Medicine and Immune Engineering, Henan University, Jinming Road, Kaifeng 475004, P.R. China

E-mail: zhaojin@henu.edu.cn

E-mail:wcjsxq@yahoo.com

Key words: mononaphthalimide-spermidine, apoptosis, mechanism designed and synthesized (4), amonafide, one representative mononaphthalimide derivative, has been selected and progressed to clinical treatment (5) but fails to enter phase III. Despite this, a series of analogues of mononaphthalimide have been evaluated in vitro $(6,7)$. UNBS5162, a new analogue of mononaphthalimide, is currently in phase I clinical trial (8). Present research also reports that linking naphthalimide with a polyamine backbone improves their cytotoxicity $(3,9,10)$ as well as selectivity against tumor cells and normal cells (11). Elinafide, a bisnaphthalimide polyamine derivative with dramatic anti-tumor activity, has been selected and progressed to clinical treatment against solid tumors $(7,12)$. Indeed, many anti-tumor drugs are poorly tumor selective resulting in high incidences of adverse effects. So, improving selective anti-tumor naphthalimide polyamine conjugations are potentially potent tumor therapeutics.

In the past decade, a series of bisnaphthalimide polyamine derivatives were synthesized and evaluated in vitro $(9,10,13,14)$. However, mononaphthalimide polyamine conjugates were seldom reported compared to their bisnaphthalimide counterparts. In our laboratory, we developed a group of mononaphthalimide polyamine conjugates with both natural and synthetic polyamine moieties exhibiting potent cytotoxic effect on several tested tumor cell lines and better cell selectivity against human hepatoma BEL-7402 $\left(\mathrm{IC}_{50}=1.06 \mu \mathrm{M}\right)$ and human normal hepatocyte QSG-7701 ( $\left.\mathrm{IC}_{50}>50 \mu \mathrm{M}\right)$, including homospermidine-mononaphthalimide (MNIHspd) conjugation and mononaphthalimide-spermidine (MNISpd) conjugate (15). Further, mononaphthalimide homospermidine conjugate was able to induce apoptosis in B16 cells (15). In fact, apoptotic mechanisms have been exploited for anti-tumor therapy as it seems to be clearly advantageous to the organism (16), and the apoptotic mechanism induced by MNISpd remains to be determined. Such information is essential in developing these types of conjugates as cytotoxic chemotherapeutics. As several cytotoxic activities of mononaphthalimide derivatives were in vitro-tested in HeLa cells and revealed different mechanisms in an agent-specific manner (4), in this study, we focused on the mechanism of action of the MNISpd-induced apoptosis in HeLa cells. 


\section{Materials and methods}

Reagents. RPMI-1640 medium and fetal calf serum (FCS) were bought from Gibco (Grand Island, NY, USA). N-acetylL-cysteine (NAC), propidium iodide (PI), dimethyl sulfoxide (DMSO), ethidium bromide (EB), acridine orange (AO) were purchased from Sigma Chemical (St. Louis, MO, USA). Caspase-3, -8, -9 Spectrophotometry Assay kit was purchased from KeyGen Biotech Co. Ltd. (Nanjing, China). Enhanced BCA protein assay kit, BeyoECL plus kit, GSH and GSSG assay kit, cell lysis buffer for Western and IP, cell mitochondria isolation kit and nuclear and cytoplasmic protein extraction kit were purchased from Beyotime Institute of Biotechnology (Jiangsu, China). Primary antibodies against B-actin, cytochrome c, Bcl-2, Bax, AIF (D-20), VDAC (voltage-dependent anion channel 1), TBP (TATA binding protein) and HRP-labeled goat anti-mouse or anti-rabbit $\lg G$ $(\mathrm{H}+\mathrm{L})$ secondary antibody were purchased from Santa Cruz Biotechnology (Santa-Cruz, CA, USA). Fluorescent dyes dichlorodihydrofluorescein diacetate (DCFH-DA) was obtained from Molecular Probes (Eugene, OR, USA). Annexin V-FITC detection kit was obtained from BD Biosciences (San Jose, CA, USA). Polyamine oxidase colorimetric assay kit was obtained from GenMed Scientifics Inc. (Arlington, USA). MNISpd was synthesised according to our previous method (15) (Fig. 1). MNISpd stock solution (10 mM) was prepared in $100 \%$ DMSO and diluted to required concentrations with FCS-free culture medium just before use. Filter-sterilized NAC was freshly prepared in FCS-free medium just before use. All other chemicals used in the experiments were commercial products of reagent grade.

Cell culture and treatments. HeLa cells (Shanghai Institutes for Biological Science, Chinese Academy of Sciences, Shanghai, China) were routinely cultured in RPMI-1640 medium supplemented with $10 \%(\mathrm{v} / \mathrm{v})$ heat-inactivated fetal calf serum, $0.2 \%$ sodium bicarbonate, $100 \mathrm{U} / \mathrm{ml}$ penicillin, $100 \mathrm{ug} / \mathrm{ml}$ streptomycin and $10 \mathrm{mM}$ HEPES. Prior to each MNISpd treatment, exponentially growing cells were grown to $80 \%$ confluence, and then aminoguanidine (final concentration: $1 \mathrm{mM}$ ) was added as an inhibitor of amine oxidase derived from FCS (17), which has no effect on the various parameters of the cells measured in this study (18). Cells that were treated with DMSO (final concentration: $0.1 \%, \mathrm{v} / \mathrm{v}$ ) in place of MNISpd were recognized as the negative control. For determining the effect of NAC, Ac-DEVD-CHO or Z-VADFMK on MNISpd-induced cytotoxicity, cells were preincubated with these reagents for $2 \mathrm{~h}$ at desired concentrations, which were also present during MNISpd treatment.

Detection of apoptosis. To determine which concentration of MNISpd was appropriate to induce HeLa cell apoptosis, Annexin V/PI assays were performed using Annexin V-FITC detection kit. Briefly, cells were treated with MNISpd (final concentration: $1,3,6,9,12,16$ and $30 \mu \mathrm{M}$ ) for $48 \mathrm{~h}$, or treated with $9 \mu \mathrm{M}$ MNISpd for 12, 24, 36, 48 and $60 \mathrm{~h}$. Cells $\left(5 \times 10^{5}\right)$ were then washed twice with cold PBS and resuspended in $500 \mu \mathrm{l}$ binding buffer. Afterward, Annexin V-FITC $(10 \mu \mathrm{l})$ was added, and incubated for $10 \mathrm{~min}$ at room temperature $(\mathrm{RT})$ followed by PI $(5 \mu \mathrm{l})$ being added and

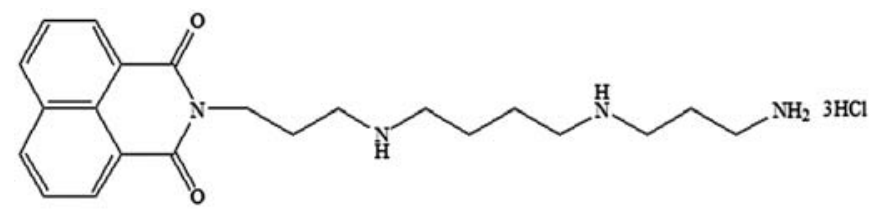

Figure 1. The structure of 2-\{3-[4-(3-Aminopropylamino)butylamino]propyl\} 1H-benz-[de]isoquino-line-1,3(2H)-dione trihydro-chloride (MNISpd).

continually incubated for $5 \mathrm{~min}$. Ten thousand cells were immediately sorted per sample in a FACSCalibur flow cytometer (Becton-Dickinson, San Jose, CA) and the data were analyzed using CellQuest software (Becton-Dickinson). Viable cells are stained by neither Annexin V-FITC nor PI (Annexin $\mathrm{V}^{-} / \mathrm{PI}^{-}$, in lower left quadrant, LL); the early apoptotic cells with intact plasma membrane are stained only by Annexin V-FITC (Annexin $\mathrm{V}^{+} / \mathrm{PI}^{-}$, in lower right quadrant, LR); necrotic cells and post-apoptotic cells are stained by both Annexin V-FITC and PI (Annexin $\mathrm{V}^{+} / \mathrm{PI}^{+}$, in upper right quadrant, UR).

To study cell plasma membrane integrity and nuclear structure, EB/AO double-staining was assessed using fluorescence microscopy (19). After treatment with MNISpd $(3,9,16$ and $30 \mu \mathrm{M})$ for $48 \mathrm{~h}$, cells were washed twice and re-suspended in PBS. Cell suspension $(200 \mu 1)$ was mixed with $8 \mu 1$ dye mixture containing the same amount of $\mathrm{AO}$ $(1 \mathrm{mg} / \mathrm{ml})$ and EB $(1 \mathrm{mg} / \mathrm{ml}$ ) (final concentration of dye mixture: $4 \mu \mathrm{g} / \mathrm{ml}$ ). MNISpd-untreated cells were recognized as the negative control. Cells treated with $0.5 \mathrm{M}$ hydrogen peroxide for $30 \mathrm{~min}$ were recognized as the positive control. Stained cells were then examined immediately under a fluorescence microscope (Olympus CKX41, Olympus, Japan) using appropriate filters.

Detection of enzymatic activation of caspase-3, -8 and -9 . Detection of enzymatic activities of caspase-3, -8 and -9 in vitro was carried out using caspase-3, -8, -9 spectrophotometry assay kit, respectively. Briefly, after treatment with MNISpd at concentration of $0,1,3,6,9,12,16$ and $30 \mu \mathrm{M}$ for $48 \mathrm{~h}$, cells were collected, washed twice with ice-cold PBS. Five million cells were re-suspended in $50 \mu 1$ of icecold lysis buffer, and then incubated on ice for $40 \mathrm{~min}$. Cell lysates were then centrifuged at $14,000 \mathrm{x}$ g for $20 \mathrm{~min}$ at $4^{\circ} \mathrm{C}$. Total protein in supernatant was quantified using Enhanced BCA protein assay kit. Each caspase substrate was added into cell extracts containing equivalent total protein, and then incubated for $4 \mathrm{~h}$ at $37^{\circ} \mathrm{C}$. Absorbance was measured at $405 \mathrm{~nm}$ using a microplate reader (Model 680, Bio-Rad, Japan).

Preparation of mitochondrial and cytosolic protein extracts. To determine the release of pro-apoptotic factors from mitochondria, mitochondrial and cytosolic protein extracts was performed using Cell Mitochondria isolation kit according to the manufacturer's instructions. Briefly, cells were treated with MNISpd or with MNISpd plus NAC at desired conditions. Then, ice-cold PBS-washed cells were re-suspended in 
2.5-ml mitochondria isolation buffer on ice for $15 \mathrm{~min}$ followed by being homogenized by 30 strokes in an ice-cold homo-genizer (Bellco Biotechnology, Vineland, NJ) until more than $50 \%$ cells were stained with trypan blue. Nonlysed cells, large plasma membrane pieces and nuclei were pelleted by centrifugation at $600 \mathrm{x}$ g for $10 \mathrm{~min}$ at $4^{\circ} \mathrm{C}$, and then supernatant was centrifuged again at 3,500 $\mathrm{x}$ g for $10 \mathrm{~min}$ at $4^{\circ} \mathrm{C}$ to obtain cytosolic fraction (supernatant) and intact mitochondria (deposition). The supernatant was further centrifuged at $12,000 \times \mathrm{g}$ for $30 \mathrm{~min}$ at $4{ }^{\circ} \mathrm{C}$ to obtain cytosolic proteins. The pellets were re-suspended in $100 \mu \mathrm{l}$ mitochondria lysis buffer, and frozen at $-70^{\circ} \mathrm{C}$ until use.

Preparation of cytoplasmic and nuclear protein extracts. To measure AIF translocation to nuclei, preparation of cytoplasmic and nuclear protein extracts was performed using Nuclear and Cytoplasmic protein extraction kit according to the manufacturer's instructions. Briefly, cells were treated with MNISpd or with MNISpd plus NAC at desired conditions, and washed with ice-cold PBS. The supernatant was carefully discarded. Then, cells were vortexed with ice-cold cytoplasmic protein extraction A buffer for $10 \mathrm{sec}$ until cells were completely scattered followed by incubation on ice for $15 \mathrm{~min}$. Cytoplasmic protein extraction buffer B was then added, and cell lysates were vortexed two times ( $5 \mathrm{sec}$ pulse, followed by $1 \mathrm{~min}$ interval) followed by being centrifuged at $14,000 \mathrm{x} \mathrm{g}$ for $5 \mathrm{~min}$ at $4^{\circ} \mathrm{C}$ to obtain supernatant (cytoplasmic proteins). Pellets were then re-suspended in nuclear protein Extraction buffer followed by being vortexed at $4^{\circ} \mathrm{C}$ for $30 \mathrm{~min}(20 \mathrm{sec}$ pulse, followed by $2 \mathrm{~min}$ interval). Cell lysates were centrifuged at $14,000 \times \mathrm{g}$ for $10 \mathrm{~min}$ at $4^{\circ} \mathrm{C}$, and supernatant was stored as nuclear protein extracts at $-70^{\circ} \mathrm{C}$ until use.

Total cellular protein extracts and Western blotting analysis. To prepare total cellular protein, MNISpd-treated cells were washed twice with ice-cold PBS and lysed using Cell lysis buffer for Western and IP kit for $30 \mathrm{~min}$ on ice. Then, cell lysates were centrifuged at $14,000 \mathrm{x} \mathrm{g}$ for $20 \mathrm{~min}$ at $4^{\circ} \mathrm{C}$. Samples at $40 \mu \mathrm{g} / \mathrm{lane}$ (total cellular protein extracts) or $20 \mu \mathrm{g} /$ lane (the other protein extracts) were separated using $12 \%$ SDS-PAGE. Proteins from the gel were transferred onto $0.45-\mu \mathrm{m}$ PVDF membrane by semi-dry transfer method using a Bio-Rad electrotransfer apparatus. The membrane was blocked with $5 \%$ non-fat milk in Tris-buffer saline supplemented with $0.05 \%$ Tween-20 (TBST, pH 7.4) at RT for $1 \mathrm{~h}$, and then incubated with primary antibodies against $B$-actin, cytochrome c, Bcl-2, Bax, AIF, VDAC, TBP overnight at $4^{\circ} \mathrm{C}$ followed by incubation with peroxidase-conjugated goat anti-mouse or anti-rabbit secondary antibody at RT for $1 \mathrm{~h}$. Membranes were washed in TBST four times for $5 \mathrm{~min}$ between each step. Protein bands were detected using BeyoECL plus.

Detection of reactive oxygen species level. Reactive oxygen species (ROS) levels in HeLa cells were measured using DCFH-DA by flow cytometry. Briefly, after treatment with MNISpd at 3, 6, 9, 12 and $16 \mu \mathrm{M}$ for 24 or $48 \mathrm{~h}$, cells were collected and washed with FCS-free medium, and then incubated with pre-warmed $\left(37^{\circ} \mathrm{C}\right)$ loading buffer (FCS-free RPMI-1640 medium containing probe to provide a final working concentration of $10 \mu \mathrm{M}$ ) at $37^{\circ} \mathrm{C}$ for $20 \mathrm{~min}$ in a $\mathrm{CO}_{2}$ incubator. Loading buffer was then discarded, and cells were washed three times with PBS to remove extracellular dyes. Cell fluorescence was analyzed by the flow cytometer in fluorescence channels $\mathrm{FL}_{1}$ using CellQuest software. Ten thousand cells were sorted per sample.

Detection of reduced and oxidized glutathione. Total glutathione (containing reduced and oxidized glutathione) level was measured using GSH and GSSG assay kit according to the manufacturer's instructions. Briefly, after treatment with MNISpd at 3, 6, 9, 12 and $16 \mu \mathrm{M}$ for 24 or $48 \mathrm{~h}$, cells were washed twice with PBS by centrifugation at $400 \mathrm{x}$ g for $5 \mathrm{~min}$ at RT. Pellets were re-suspended in protein removal buffer and vortexed immediately followed by being lysed by two freeze-thaw cycles. After cell lysates were centrifuged at $10,000 \times \mathrm{g}$ for $10 \mathrm{~min}$ at $4^{\circ} \mathrm{C}$, supernatant containing total glutathione was transferred to another tube. For quantifying total glutathione level, $10 \mu 1$ supernatant was mixed with $150 \mu 1$ total glutathione assay buffer at RT for $5 \mathrm{~min}$, and then $50 \mu 1 \mathrm{NADPH}(0.16 \mathrm{mg} / \mathrm{ml})$ was added. Absorbance was read at $405 \mathrm{~nm}$ using the microplate reader. Oxidized form glutathione (GSSG) level was quantified using the same method for total glutathione assay after supernatant was pretreated with $1 \% 1 \mathrm{M} 2$-vinylpyridine solution at RT for $60 \mathrm{~min}$ to remove reduced form glutathione (GSH) from total glutathione. The amount of GSH was obtained by subtracting GSSG from total glutathione.

Detection of PAO activity. The activity of polyamine oxidase (PAO, EC.1.5.3.11) was assayed using Polyamine oxidase colorimetric assay kit. In this assay, oxidation of $\mathrm{N}^{1}$-acetylspermine by PAO produced the spermine-dependent formation of hydrogen peroxide. Peroxide release was detected by measuring the production of oxidized o-dianisidine, which can be detected by its absorbance at $440 \mathrm{~nm}$. According to the manufacturer's instructions, after cells were treated with MNISpd (final concentration: 3, 6, 9, 12 and $16 \mu \mathrm{M}$ ) for $24 \mathrm{~h}$, or treated with $9 \mu \mathrm{M}$ MNISpd for $12,24,36$ and $48 \mathrm{~h}$, cells $\left(5 \times 10^{6}\right)$ were washed twice with PBS, and then lysed in $500-\mu 1$ lysis buffer on ice for $30 \mathrm{~min}$. Cell lysates were centrifuged at $16,000 \mathrm{x}$ g for $5 \mathrm{~min}$ at $4^{\circ} \mathrm{C}$. Supernatant $(50 \mu 1$, containing $50 \mu \mathrm{g}$ proteins) was added into 800- $\mu 1$ assay buffer in an acuvette in the dark followed by incubation with $100 \mu \mathrm{l}$ probe for $2 \mathrm{~min}$ at $37^{\circ} \mathrm{C}$. $\mathrm{N}^{1}$-acetylspermine $(50 \mu \mathrm{l})$ was then added. The data were immediately read at 0 and $5 \mathrm{~min}$ (time intervals for $5 \mathrm{~min}$ ) at $440 \mathrm{~nm}$ in a spectrophotometer. PAO activity was defined as: one unit would oxidize $1.0 \mu \mathrm{M}$ of $\mathrm{N}^{1}$-acetyl-spermine to 3 -aminopropanal and $\mathrm{H}_{2} \mathrm{O}_{2}$ per minute at $\mathrm{pH} 8.0$ at $37^{\circ} \mathrm{C}$.

Statistical analysis. A minimum of three independent experiments were carried out for each assay. Statistics were performed with the SPSS statistics program (windows version 11.0). Data are expressed as the means \pm S.D. To evaluate whether observed differences were significant, Student's t-test for comparisons between two groups was used; or oneway ANOVA with Tukey post-hoc test for comparisons among three or more groups was used. $\mathrm{P}<0.05$ was considered to be significant for all analyses. 

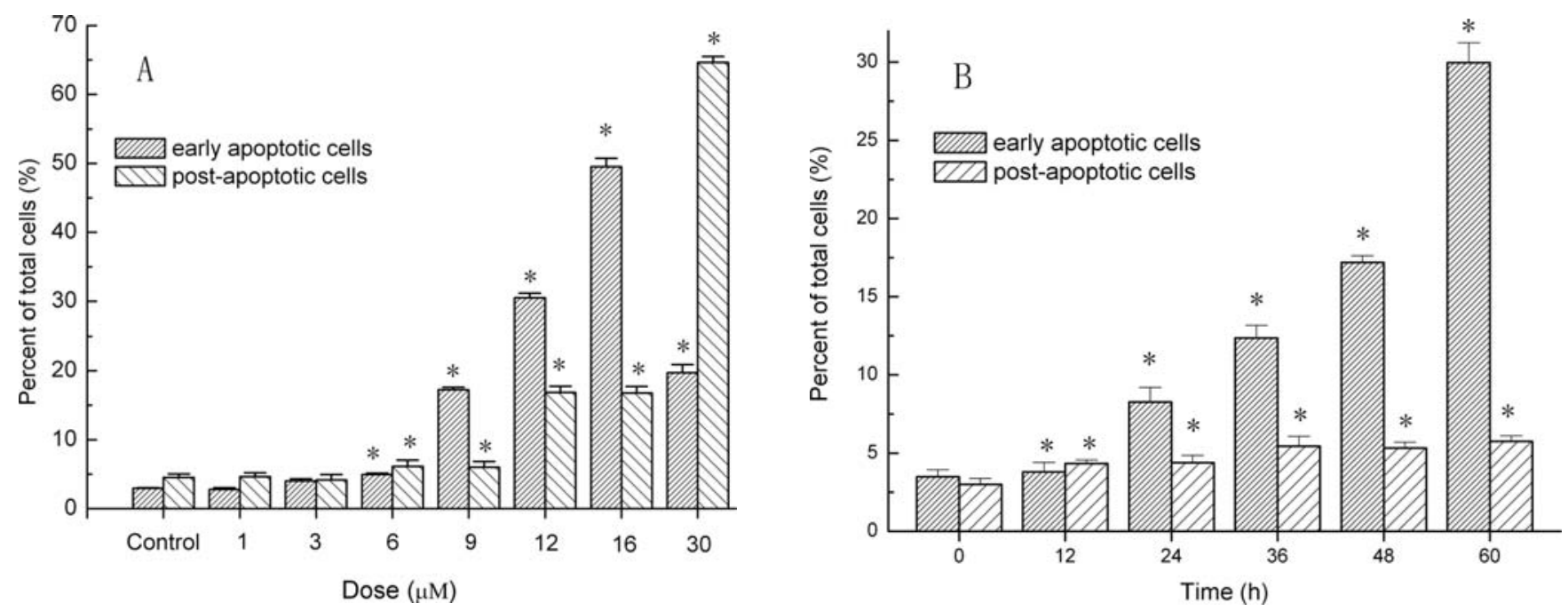

Figure 2. MNISpd-induced apoptosis in HeLa cells was measured by Annexin V/PI assay by a flow cytometer. (A) Cells were treated with MNISpd at different concentrations (1-30 $\mu \mathrm{M})$ for $48 \mathrm{~h}$. MNISpd-untreated cells were recognized as the negative control. (B) Cells were treated with MNISpd at $9 \mu \mathrm{M}$ for different time $(12,24,36,48$ and $60 \mathrm{~h})$. MNISpd-untreated cells for $24 \mathrm{~h}$ were recognized as the negative control. Each value represents the means \pm S.D. of at least three independent experiments. ${ }^{*} \mathrm{P}<0.05$ (MNISpd treatment vs. Cont.).
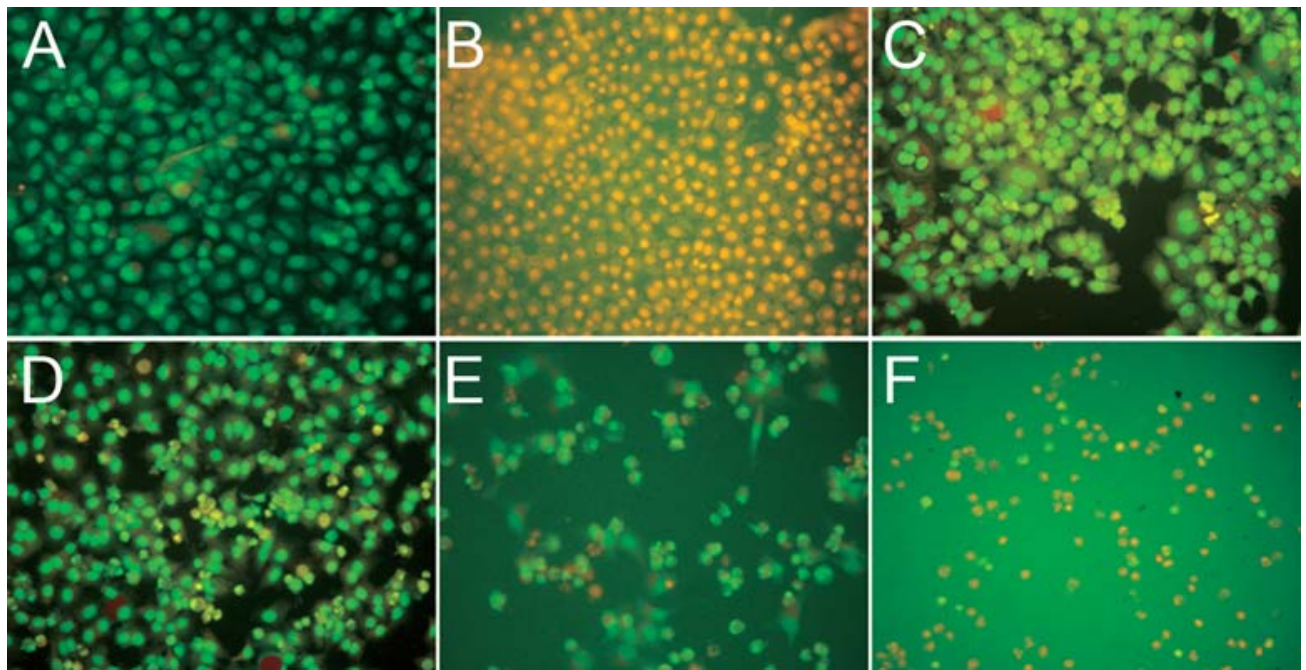

Figure 3. Cell membrane integrity and nuclear structure in HeLa cells were assessed by EB/AO double-staining (magnification, x200). (A) Cells without MNISpd treatment which have a uniform green color in their nuclei were recognized as living cells. (B) Cells treated with $0.5 \mathrm{M}$ hydrogen peroxide for 30 min which have a uniform bright orange chromatin with organized structure were as the necrotic death control. (C-F) Cells were treated with MNISpd at different concentrations: 3, 9, 12 and $30 \mu \mathrm{M}$ for $48 \mathrm{~h}$, respectively. Each experiment was performed at least three times and the results were consistent.

\section{Results}

Effect of MNISpd on apoptosis. As shown in Fig. 2A and B, early- and post-apoptotic ratios increased in a time- and dosedependent manner. Furthermore, the differences in apoptotic ratio between 9- and 6- $\mu \mathrm{M}$ MNISpd treatment were significant. Post-apoptotic ratio significantly increased after cells were treated with MNISpd $(\geq 12 \mu \mathrm{M})$ for $48 \mathrm{~h}$ compared with those cells treated with $9 \mu \mathrm{M}$. However, a lower post-apoptotic ratio was induced by $9-\mu \mathrm{M}$ MNISpd treatment during a $60-\mathrm{h}$ period. As shown in Fig. 3, the cells in the negative control showed normal structures (Fig. 3A). The cells treated with $0.5 \mathrm{M} \mathrm{H}_{2} \mathrm{O}_{2}$ for $30 \mathrm{~min}$ were stained with uniform chromatin and cells swelled simultaneously (Fig. 3B). After treatment with $3 \mu \mathrm{M}$ MNISpd (Fig. 3C), cells rarely showed shrinkage, membrane blebbing or chromatin condensation. The cells treated with MNISpd at 9 and $12 \mu \mathrm{M}$ showed chromatin condensation, apoptotic-like nuclei fragmentation (Fig. 3D and E), and most cells exhibited nucleus fragment, apoptotic bodies and decreased cell volume (Fig. 3F) with $30-\mu \mathrm{M}$ MNISpd treatment.

Exposure to MNISpd ( $\geq 3 \mu \mathrm{M}$ for $48 \mathrm{~h}$ ) led to a significant and dose-dependent increase in activation of caspase-3, -9 but not caspase-8 in HeLa cells (Fig. 4). Although, the activity of caspase-3, or both activity of caspase-3 and caspase- 9 were completely inhibited by Ac-DEVD-CHO or Z-VADFMK pre-incubation, respectively (revealed by spectrophotometry assay, data not shown), these two inhibitors only partly 


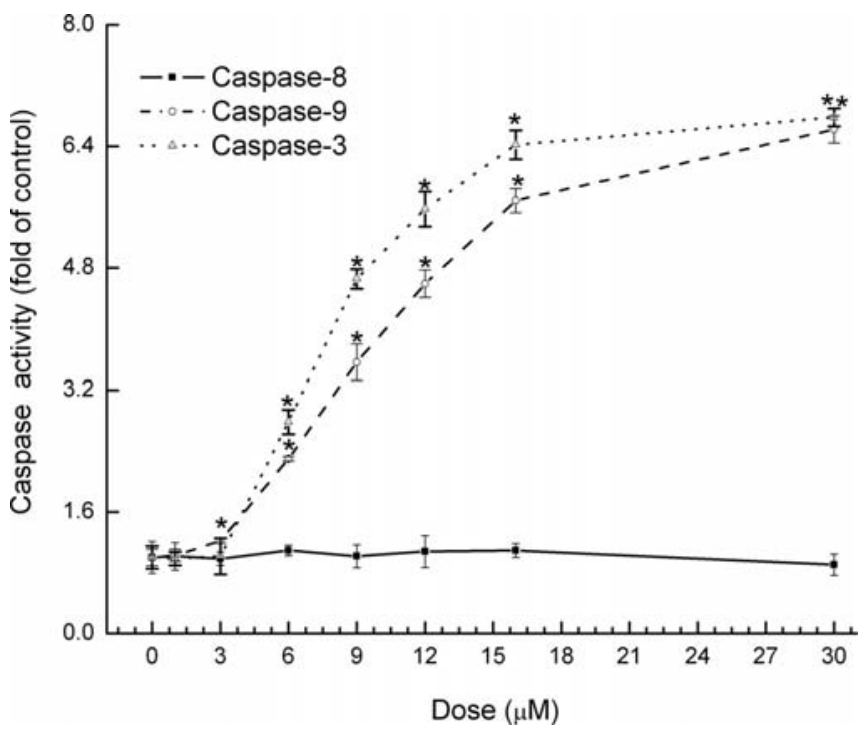

Figure 4. Variations in caspase activities of HeLa cells were measured by spectrophotometry. Total protein extracts were obtained from MNISpduntreated cells (negative control) and the cells that were treated with MNISpd at concentrations of 1, 3, 6, 9, 12, 16 and $30 \mu \mathrm{M}$ for $48 \mathrm{~h}$. Tota protein extracts $(20 \mu \mathrm{g} / \mathrm{sample})$ were used to determine caspase- $9,-8$ and -3 activities. Each value represents the means \pm S.D. of at least three independent experiments. ${ }^{*} \mathrm{P}<0.05$ (MNISpd treatment vs. Cont.).

increased the survival percentage of MNISpd-treated cells, but did not completely protect HeLa cells against the MNISpd-induced apoptosis (Fig. 5).

Effect of MNISpd on mitochondria-related events. Western blotting experiments showed that MNISpd induced a

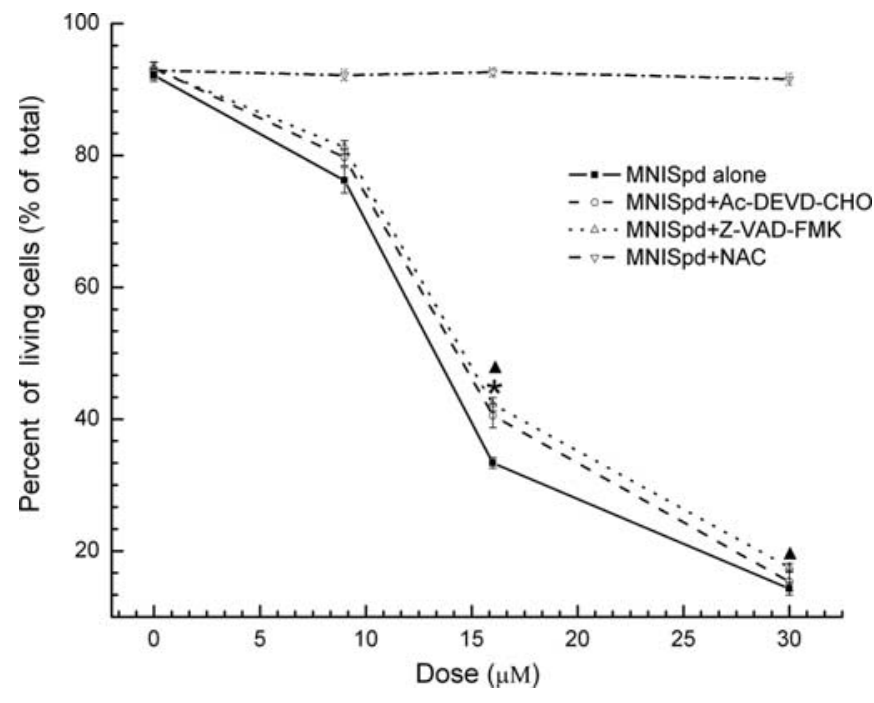

Figure 5. HeLa cell survival was measured by Annexin V/PI assay. The plot presents the percentage of cells located in the lower left quadrant derived from flow-cytometric analysis. Cells were treated with MNISpd (9, 16 and $30 \mu \mathrm{M})$ alone for $48 \mathrm{~h}$, or treated with MNISpd $(9,16$ and $30 \mu \mathrm{M})$ for $48 \mathrm{~h}$ after Ac-DEVD-CHO $(50 \mu \mathrm{M})$, Z-VAD-FMK $(50 \mu \mathrm{M})$ or NAC $(10 \mathrm{mM})$ pre-incubation for $2 \mathrm{~h}$. Each value represents the means \pm S.D. of at least three independent experiments. ${ }^{*} \mathrm{P}<0.05$ (MNISpd plus Ac-DEVD-CHO treatment vs. MNISpd alone treatment). Triangles indicate $\mathrm{P}<0.05$ (MNISpd plus Z-VAD-FMK treatment vs. MNISpd alone treatment).

cytochrome $\mathrm{c}$ increase in the cytosol concomitant with a cytochrome c decrease in the mitochondria (Fig. 6A). With MNISpd $(9 \mu \mathrm{M})$ treatment (Fig. 6B), AIF was transferred from mitochondria via cytoplasm to nucleus over a 48-h period. After treatment with MNISpd for $48 \mathrm{~h}$ (Fig. 6C), the expression of Bax protein was up-regulated, the Bcl-2 level
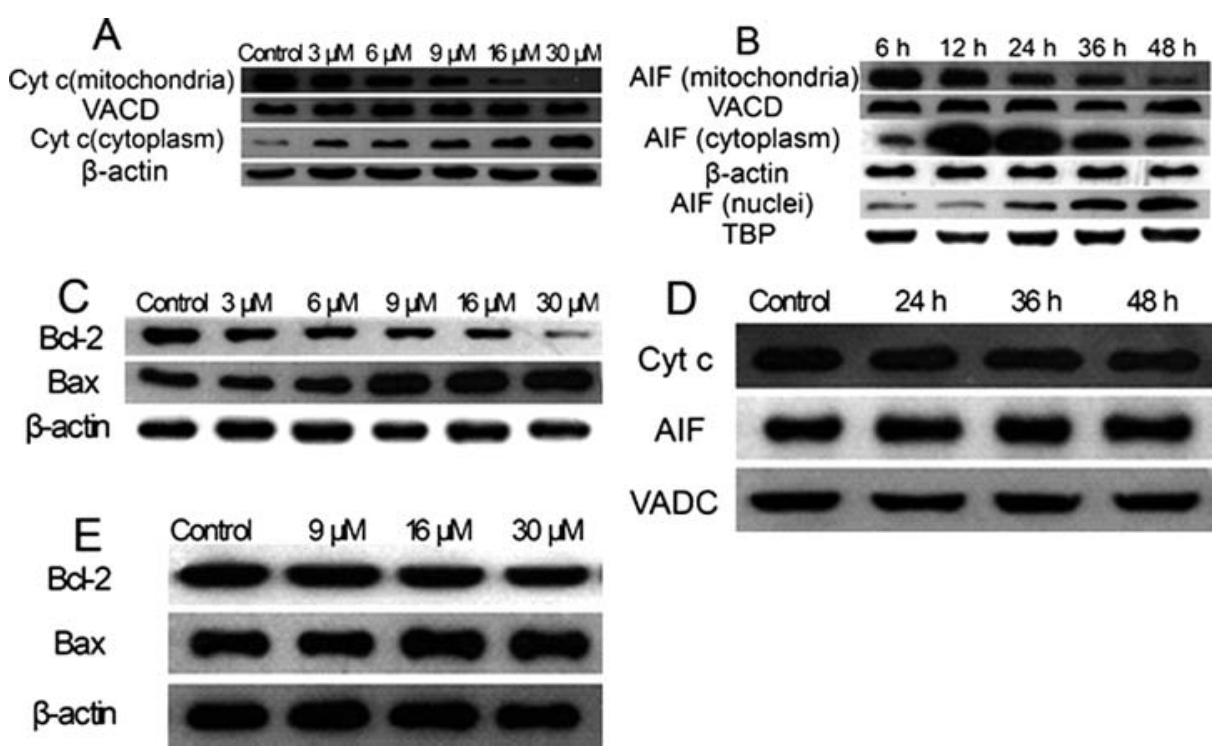

Figure 6. Expressions of mitochondrial apoptosis pathway-related proteins in HeLa cells were measured by Western blot analysis. B-actin, VDAC and TBP were used as loading controls for cytoplasm, mitochondria and nuclei fractions, respectively. (A) Cells were treated with MNISpd at different concentrations $(3,6,9,16$ and $30 \mu \mathrm{M})$ for $48 \mathrm{~h}$. Levels of cytochrome c were determined using cytosolic and mitochondrial extracts. (B) Cells were treated with $9 \mu \mathrm{M}$ of MNISpd at desired time (12, 24, 36 and $48 \mathrm{~h}$ ), and then AIF levels in cytoplasm, mitochondria and nuclei were detected. (C) Cells were treated with MNISpd at desired concentrations $(3,6,9,16$ and $30 \mu \mathrm{M}$ ) for $48 \mathrm{~h}$. Expressions of Bcl-2, and Bax were measured using total protein extracts. (D) Cells were treated with MNISpd at $9 \mu \mathrm{M}$ for 24, 36 and $48 \mathrm{~h}$ after pre-incubation with $10 \mathrm{mM}$ NAC for $2 \mathrm{~h}$. Levels of cytochrome c and AIF in mitochondria of HeLa cells were measured. (E) Cells were treated with MNISpd at desired concentrations (9, 16 and $30 \mu \mathrm{M})$ for $48 \mathrm{~h}$ after pre-incubation with $10 \mathrm{mM}$ NAC for $2 \mathrm{~h}$. Expressions of Bcl-2 and Bax were measured using total protein extracts. Typical images are shown. Each experiment was performed at least three times and results were consistent. 


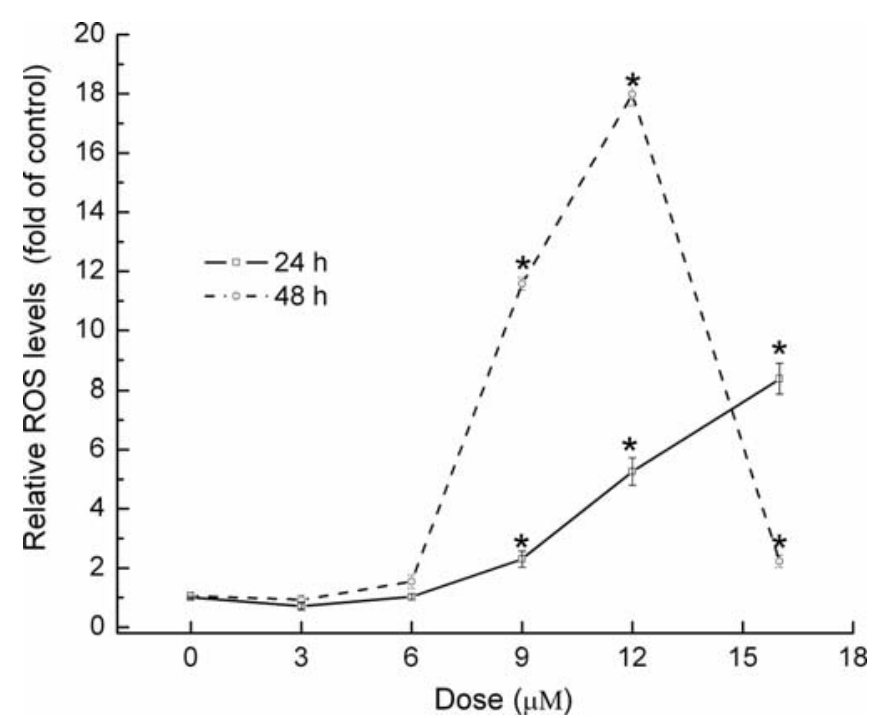

Figure 7. MNISpd-induced ROS accumulation in HeLa cells was measured using DCFH-DA by a flow cytometer. Cells were treated with MNISpd at desired concentrations $(3,6,9,12$ and $16 \mu \mathrm{M})$ for 24 or $48 \mathrm{~h}$, and then loaded with DCFH-DA. ${ }^{*} \mathrm{P}<0.05$ (MNISpd treatment vs. Cont.). Each value represents the means \pm S.D. of at least three independent experiments.

was down-regulated in a dose-dependent manner. On the other hand, NAC pre-incubation could prevent the release of cytochrome $\mathrm{c}$ and AIF out of mitochondria (Fig. 6D) as well as the variations in Bax and Bcl-2 expression (Fig. 6E).

Effect of MNISpd on ROS generation and intracellular glutathione level. During a 24 -h period, a slight decrease following a slight increase in intracellular ROS level were observed with 3- and 6- $\mu \mathrm{M}$ MNISpd treatments, respectively, but not significant. Then, intracellular ROS level significantly increased with 9-, 12- and 16- $\mu \mathrm{M}$ MNISpd treatment in a dose-dependent manner (Fig. 7). Variations in ROS level with MNISpd treatment for $48 \mathrm{~h}$ revealed a similar dosedependent trend, and a peak of ROS level was observed with $12-\mu \mathrm{M}$ MNISpd treatment followed by a significantly decreased ROS level with 16- $\mu \mathrm{M}$ MNISpd treatments.

The size of total glutathione pool primarily increased in MNISpd (3 and $6 \mu \mathrm{M}$ )-treated cells, and then decreased rapidly in those cells treated with MNISpd ranging from 9 to $16 \mu \mathrm{M}$ (Fig. 8A). A sustained decrease in the ratio of GSH to GSSG in HeLa cells was observed with MNISpd treatment ranging from 3 to $16 \mu \mathrm{M}$ in a dose-dependent manner during a 48-h period (Fig. 8B).

Effect of MNISpd on PAO induction. After HeLa cells were treated with MNISpd ranging from 3 to $16 \mu \mathrm{M}$ for $24 \mathrm{~h}$, or treated with MNISpd at $9 \mu \mathrm{M}$ during a 48 -h period, PAO activity was up-regulated in a time- and dose-dependent manner (Fig. 9A and B).

\section{Discussion}

Polyamine derivatives are known as apoptosis inducers (20), and naphthalimide derivatives-induced DNA damage has been widely studied $(4,6,7)$. Many naphthalimide polyamine conjugates have been synthesized as a new type of selective anti-tumor agents. It is reported that bisnaphthalimidopropyl spermidine (BNIPSpd) induces apoptosis within promyelocytic leukaemia cells (HL60) (21) as well as colon carcinoma cells (Caco-2 and HT-29) exhibiting DNA fragmentation, elevated caspase-3 activity and apoptotic bodies (10). MNIHspd was able to kill B16 cells via both mitochondrial and cellular membrane death receptor apoptosis pathways (15), and another naphthalimidepolyamine conjugate induced apoptosis in hepatoma HepG2 cells via a mitochondrial pathway (22). These reports indicate that naphthalimide polyamine conjugates exhibit
A

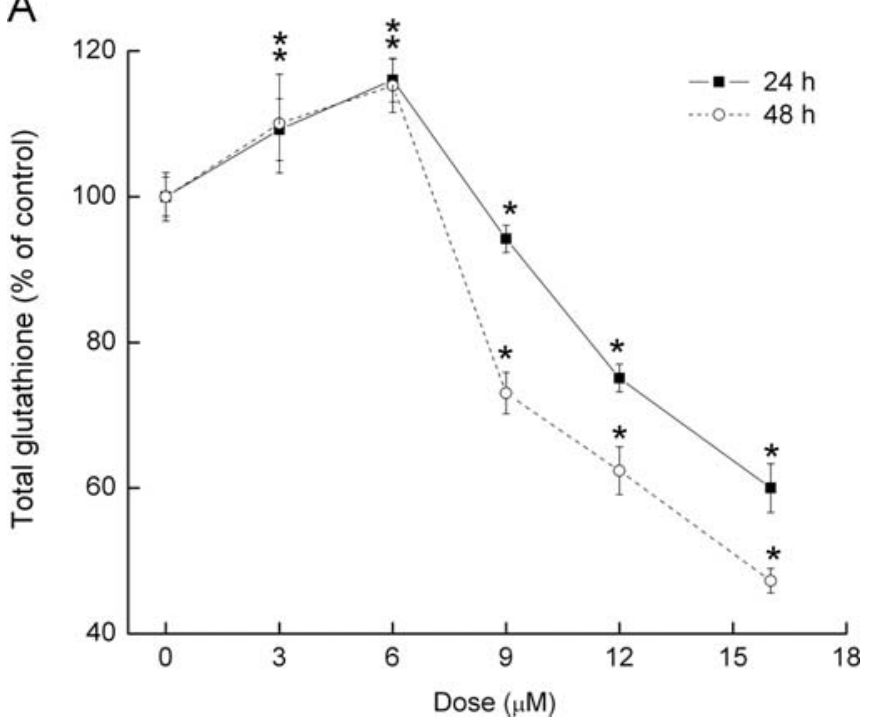

B

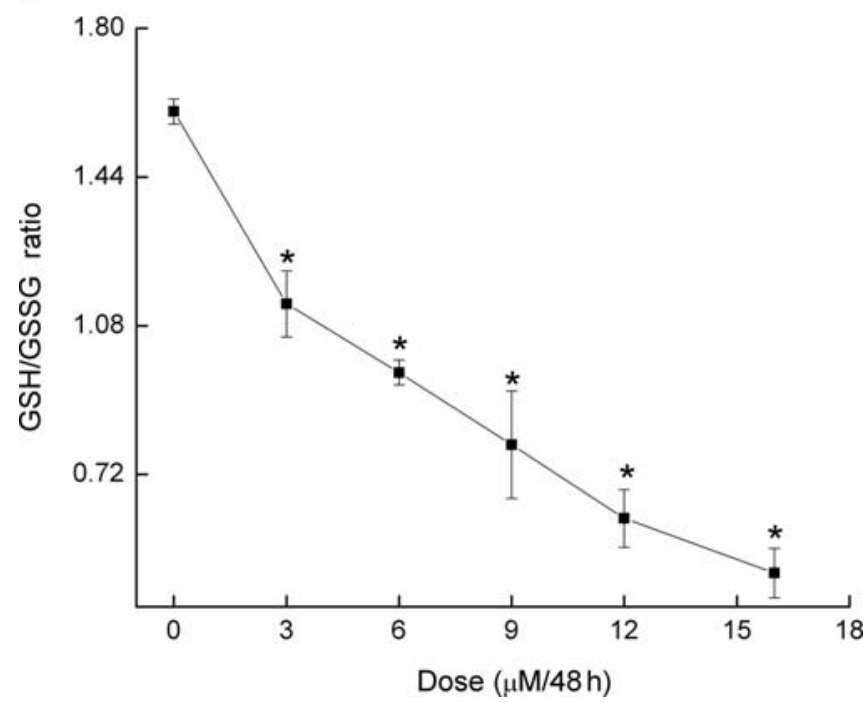

Figure 8. Intracellular variations in total glutathione levels and ratio of GSH to GSSG in HeLa cells were measured by spectrophotometry. Cells were treated with MNISpd at a range from 3 to $16 \mu \mathrm{M}$ for 24 or $48 \mathrm{~h}$, and then intracellular glutathione content was measured. (A) Total glutathione was expressed relative to the control. (B) Ratio of GSH to GSSG was expressed. ${ }^{*} \mathrm{P}<0.05$ (MNISpd treatment vs. Cont.). Each value represents the means \pm S.D. of at least three independent experiments. 

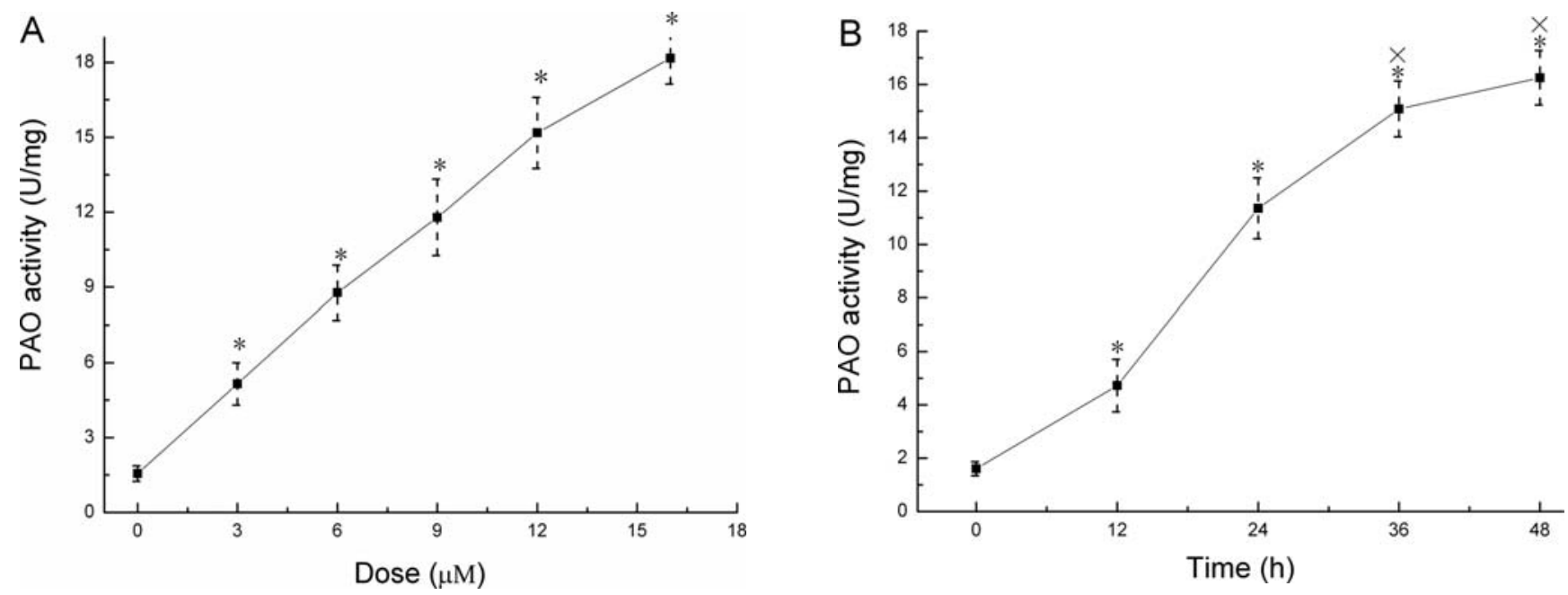

Figure 9. Variations in PAO activities of HeLa cells were measured by spectrophotometry. Total protein extracts were used to determine PAO activities. MNISpd-untreated cells were recognized as the negative control. (A) Cells were treated with MNISpd at concentrations of 3, $6,9,12$ and $16 \mu \mathrm{M}$ for $24 \mathrm{~h}$. ${ }^{*} \mathrm{P}<0.05$ (MNISpd treatment vs. Cont.). (B) Cells were treated with MNISpd at $9 \mu \mathrm{M}$ for 12, 24, 36 and $48 \mathrm{~h}$. ${ }^{*} \mathrm{P}<0.05$ (MNISpd treatment vs. Cont.). Crosses indicate $\mathrm{P}>0.05$ (MNISpd treatment for $36 \mathrm{~h}$ vs. MNISpd treatment for $48 \mathrm{~h}$ ). Each value represents the means \pm S.D. of at least three independent experiments.

different apoptotic mechanisms in an agent- and cell-typespecific manner. However, only a few reports on the apoptotic mechanism of action of naphthalimide polyamine conjugates are reported. Thus, it is necessary to accumulate more comprehensive data to discover exact apoptotic mechanisms of these conjugates.

Apoptosis is characterized by specific biochemical features such as exposing PS to external cellular environment, activation of caspase, cell shrinkage, membrane blebbing and chromatin condensation. MNISpd-induced apoptosis in HeLa cells was confirmed by Annexin V/PI and EB/AO doublestaining assays. Furthermore, based on our data resulting from Annexin V/PI assays, it indicated that $9 \mu \mathrm{M}$ MNISpd induced apoptosis, which seldom induced necrosis or postapoptosis in HeLa cells during a $60-\mathrm{h}$ period. Thus, in some studies on the action of mechanism of apoptosis induced by MNISpd in this work, 9- $\mu \mathrm{M}$ MNISpd treatments were used.

Previous reports indicate polyamine derivatives-induced apoptosis via caspase-dependent pathway (11), and rarely polyamine and naphthalimide derivatives, also naphthalimide-polyamine conjugations showed an induction of apoptosis via caspase-independent pathway (23). Our data resulting from caspase activating, cytochrome c release and caspase inhibitor assays indicated that MNISpd-induced apoptosis in HeLa cells not only sensitive to the intrinsic caspase-dependent pathway without apparent participation of the extrinsic caspase-dependent pathway but also caspaseindependent cell death.

AIF release from mitochondria has been observed in several instances of caspase-independent cell death in response to a variety of death stimuli $(24,25)$. Importantly, AIF effects are not inhibited by pharmacological caspase inhibitors such as Z-VAD-FMK $(26,27)$. Our findings for the first time confirmed the AIF translocation from mitochondria to the nucleus, indicating that MNISpd-induced apoptosis was not only correlated to intrinsic caspase-dependent pathway but also AIF-related caspase-independent apoptotic pathway.
Mitochondria-mediated apoptosis is usually regulated by Bcl-2 family proteins such as Bax, a pro-apoptotic protein known to induce mitochondrial membrane permeability and lead to a release of apoptosis-related factors from the mitochondria to cytosol (28), and Bcl-2, an anti-apoptotic member known to preserve mitochondrial integrity that elicits opposing effects on Bax. Some research indicates a relationship between Bcl-2 family and polyamine conjugation-induced apoptosis $(29,30)$. Recently, a downregulation of $\mathrm{Bcl}-2$, p-Bad and $\mathrm{Bcl}-\mathrm{xL}$ in $\mathrm{HepG} 2$ cells with naphthalimide-polyamine conjugate treatment was reported (21). Our studies indicated that apoptosis regulation in MNISpd-treated HeLa cells was related to Bcl-2/Bax mitochondria-dependently.

The balance between oxidant production and antioxidant is importance to maintain cells survival. Reactive oxygen species including free radicals such as superoxide, hydroxyl radical and non-radical derivatives of oxygen has been shown to be highly conserved signals to trigger cell death (31). GSH is the main antioxidant system in cells, which protects cells against the damage resulting from oxidative stress through eliminating ROS. In contrast, strong oxidative stress could deplete intracellular GSH $(32,33)$, causing cell death. ROS, resulting from the catabolic oxidation of polyamines, has been confirmed to be linked to the cytotoxicity of anti-tumor polyamine analogues (34). However, the generation of ROS induced by polyamine derivatives may be both agent and cell-type specific. The evaluation of the relationship between ROS production and polyamine derivative treatment is not comprehensive. Our findings resulting from DCFH-DA staining assay indicated a significant ROS accumulation in $>9 \mu \mathrm{M}$ MNISpd-treated HeLa cells, at the same time, total glutathione pool in HeLa cells was switched from initial up-regulation followed by down-regulation following the increase of MNISpd concentration. Based on these data, it indicated that intracellular ROS accumulation was related to the size of GSH pool in HeLa cells in accordance with a theory that GSH depletion facilitates the 
accumulation of ROS in anti-tumor drugs-treated cells (35). Thus, it was further deduced that a small quantity of ROS generated by MNISpd treatment $(\leq 6 \mu \mathrm{M})$ increased the size of total glutathione pool (containing reduced and oxidized glutathione) in HeLa cells to eliminate the ROS for maintaining the normal intracellular redox station; a large quantity of ROS generated by MNISpd treatment $(\geq 9 \mu \mathrm{M})$ consumed the GSH pool leading to an accumulation of ROS in turn. Corresponding to this process, ROS levels initially not significantly increased with MNISpd treatment $(\leq 6 \mu \mathrm{M})$, and then rapidly increased with MNISpd treatment $(9,12$ and $16 \mu \mathrm{M})$ in a time-dependent manner during a 24-h period, which in turn increased the lethality of MNISpd resulting in a dramatical decline of ROS levels with $16-\mu \mathrm{M}$ MNISpd treatment for $48 \mathrm{~h}$ (Fig. 7), as dead cells will not load with DCFH-DA.

NAC, which was widely used as a precursor of GSH to eliminate ROS, has been reported to protect cells against oxidative stress-induced cell death (36). In order to verify the relationship between ROS and MNISpd-induced apoptosis, a series of assays, using NAC as a ROS elimination agent, were performed. Pre-incubation with $10 \mathrm{mM}$ NAC for $2 \mathrm{~h}$ completely inhibited the apoptosis induced by $30-\mu \mathrm{M}$ MNISpd treatment for $48 \mathrm{~h}$, revealed by Annexin V/PI assay with an apoptotic ratio (containing all cells that were in LR and UR) of $6.09 \% \pm 1.32$. However, pre-incubation with 5 or $20 \mathrm{mM}$ NAC for $2 \mathrm{~h}$, the apoptotic ratio (containing all cells that were in LR and UR) induced by $30-\mu \mathrm{M}$ MNISpd treatment for $48 \mathrm{~h}$ were $26.59 \% \pm 1.83$ and $14.19 \% \pm 1.87$, respectively, indicating that $10 \mathrm{mM}$ NAC was suitable to prevent HeLa cells from ROS-mediated apoptosis. Combined with the findings of cytochrome $\mathrm{c}$ and AIF release out of mitochondria and the variations in $\mathrm{Bax}$ and $\mathrm{Bcl}-2$ expression being antagonized by $10 \mathrm{mM}$ NAC, it indicated that MNISpd-induced apoptosis was correlated to intracellular ROS accumulation, and mitochondria damage played an important role in the MNISpd-induced apoptosis.

In polyamine metabolism retroconversion process, using $\mathrm{N}^{1}$-acetyl polyamine derivatives as substrates, $\mathrm{PAO}$ produces spermidine and putrescine following production of ROS, particularly $\mathrm{H}_{2} \mathrm{O}_{2}$, as a by-product (37). The asymmetrically substituted polyamine derivatives can increase PAO activity, which is the source of ROS in turn contributing to apoptosis induction of polyamine analogues $(38,39)$. Our finding confirmed a significantly increase in PAO activity in MNISpdtreated HeLa cells. Thus, MNISpd-induced ROS production may be related to PAO activation. It is noteworthy that PAO is likely to contribute to the polyamine analogue resistance in some specific cells, as high levels of PAO have a capacity to detoxify certain symmetrically and asymmetrically substituted polyamine analogues $(40,41)$. Therefore, this capacity is a possible reason why MNISpd cytotoxicity is less than other naphthalimide derivatives.

In conclusion, MNISpd treatment $(\geq 6 \mu \mathrm{M})$ induced apoptosis in HeLa cells via caspase-dependent intrinsic and AIF-mediated caspase-independent pathways relating to sustaining intracellular ROS accumulation, and $9 \mu \mathrm{M}$ is an appropriate concentration to induce HeLa cell apoptosis. MNISpd-induced ROS accumulation is correlated to GSH pool depletion. MNISpd up-regulates the activity of PAO, which is likely to be the source of ROS.

\section{Acknowledgements}

This research was supported by the Major Research plan of the National Natural Science Foundation of China (grant no. 90913001) and Henan Key Project of Science and Technology (082102270002) for Chaojie Wang. This research was also supported by Henan Provincial College Young Backbone Teachers foundation Project for Lianhe Yang. The authors wish to thank Professor Bo Peng, the Director of Key Laboratory for AIDS Research of State Administration of Traditional Chinese Medicine, for providing laboratory and helpful advice. The authors wish to thank Mr. Songcan Liu (Henan College of Traditional Chinese Medicine, ZhengZhou, China) for their excellent assistance to the experimental work.

\section{References}

1. Wu A, Xu Y, Qian X, Wang J and Liu J: Novel naphthalimide derivatives as potential apoptosis-inducing agents: design, synthesis and biological evaluation. Eur J Med Chem 44: 4674-4680, 2009.

2. Van Quaquebeke E, Mahieu T, Dumont P, et al: 2,2,2Trichloro-N-(\{2-[2-(dimethy-lamino)-ethyl]-1,3-dioxo-2,3dihydro-1H-benzo[de]isoquinolin-5-yl\}carbamoyl)acetamide (UNBS3157), a novel nonhematotoxic naphthalimide derivative with potent antitumor activity. J Med Chem 50: 4122-4134, 2007.

3. Braña MF, Castellano JM, Perron D, et al: Chromophoremodified bis-naphthalimides: synthesis and antitumor activity of bis-dibenz[de,h]isoquinoline-1,3-diones. J Med Chem 40: 449-454, 1997

4. Ingrassia L, Lefranc F, Kiss R and Mijatovic T: Naphthalimides and azonafides as promising anti-cancer agents. Curr Med Chem 16: 1192-1213, 2009.

5. Malviya VK, Liu PY, Alberts DS, Surwit EA, Craig JB and Hannigan EV: Evaluation of amonafide in cervical cancer, phase II. A SWOG study. Am J Clin Oncol 15: 41-44, 1992.

6. Braña MF, Cacho M, Garcia MA, et al: New analogues of amonafide and elinafide, containing aromatic heterocycles: synthesis, antitumor activity, molecular modeling, and DNA binding properties. J Med Chem 47: 1391-1399, 2004.

7. Braña MF, Cacho M, Ramos A, et al: Synthesis, biological evaluation and DNA binding properties of novel mono and bisnaphthalimides. Org Biomol Chem 1: 648-654, 2003.

8. Mijatovic T, Mahieu T, Bruyere C, et al: UNBS5162, a novel naphthalimide that decreases CXCL chemokine expression in experimental prostate cancers. Neoplasia 10: 573-586, 2008.

9. Dance AM, Ralton L, Fuller Z, et al: Synthesis and biological activities of bisnaphthalimido polyamines derivatives: cytotoxicity, DNA binding, DNA damage and drug localization in breast cancer MCF 7 cells. Biochem Pharmacol 69: 19-27, 2005.

10. Ralton LD, Bestwick CS, Milne L, Duthie S and Kong Thoo Lin P: Bisnaphthalimidopropyl spermidine induces apoptosis within colon carcinoma cells. Chem Biol Interact 177: 1-6, 2009.

11. Xie S, Wang J, Zhang Y and Wang C: Antitumor conjugates with polyamine vectors and their molecular mechanisms. Expert Opin Drug Deliv 7: 1049-1061, 2010.

12. Villalona-Calero MA, Eder JP, Toppmeyer DL, et al: Phase I and pharmacokinetic study of LU79553, a DNA intercalating bisnaphthalimide, in patients with solid malignancies. J Clin Oncol 19: 857-869, 2001.

13. Pavlov V, Kong Thoo Lin P and Rodilla V: Cytotoxicity, DNA binding and localisation of novel bis-naphthalimidopropyl polyamine derivatives. Chem Biol Interact 137: 15-24, 2001.

14. Oliveira J, Ralton L, Tavares J, et al: The synthesis and the in vitro cytotoxicity studies of bisnaphthalimidopropyl polyamine derivatives against colon cancer cells and parasite Leishmania infantum. Bioorg Med Chem 15: 541-545, 2007.

15. Tian ZY, Xie SQ, Du YW, et al: Synthesis, cytotoxicity and apoptosis of naphthalimide polyamine conjugates as antitumor agents. Eur J Med Chem 44: 393-399, 2009.

16. Fulda $\mathrm{S}$ and Debatin KM: Exploiting death receptor signaling pathways for tumor therapy. Biochim Biophys Acta 1705: 27-41, 2004. 
17. Monti MG, Ghiaroni S, Pernecco L, Barbieri D, Marverti G and Franceschi C: Polyamine depletion protects HL-60 cells from 2-deoxy-D-ribose-induced apoptosis. Life Sci 62: 799-806, 1998.

18. Xie SQ, Liu GC, Ma YF, et al: Synergistic antitumor effects of anthracenylmethyl homospermidine and alpha-difluoromethylornithine on promyelocytic leukemia HL60 cells. Toxicol In Vitro 22: 352-358, 2008.

19. McGahon AJ, Martin SJ, Bissonnette RP, et al: The end of the (cell) line: methods for the study of apoptosis in vitro. Methods Cell Biol 46: 153-185, 1995.

20. Casero RA Jr and Woster PM: Recent advances in the development of polyamine analogues as antitumor agents. J Med Chem 52: 4551-4573, 2009 .

21. Kong Thoo Lin P, Dance AM, Bestwick C and Milne L: The biological activities of new polyamine derivatives as potential therapeutic agents. Biochem Soc Trans 31: 407-410, 2003.

22. Tian ZY, Xie SQ, Mei ZH, Zhao J, Gao WY and Wang CJ: Conjugation of substituted naphthalimides to polyamines as cytotoxic agents targeting the Akt/mTOR signal pathway. Org Biomol Chem 7: 4651-4660, 2009.

23. Ha HC, Woster PM and Casero RA Jr: Unsymmetrically substituted polyamine analogue induces caspase-independent programmed cell death in Bcl-2-overexpressing cells. Cancer Res 58: 2711-2714, 1998.

24. Daugas E, Nochy D, Ravagnan L, et al: Apoptosis-inducing factor (AIF): a ubiquitous mitochondrial oxidoreductase involved in apoptosis. FEBS Lett 476: 118-123, 2000.

25. Thayyullathil F, Chathoth S, Hago A, Patel M and Galadari S: Rapid reactive oxygen species (ROS) generation induced by curcumin leads to caspase-dependent and -independent apoptosis in L929 cells. Free Radic Biol Med 45: 1403-1412, 2008.

26. Cande C, Vahsen N, Garrido C and Kroemer G: Apoptosisinducing factor (AIF): caspase-independent after all. Cell Death Differ 11: 591-595, 2004.

27. Susin SA, Daugas E, Ravagnan L, et al: Two distinct pathways leading to nuclear apoptosis. J Exp Med 192: 571-580, 2000.

28. Gross A, McDonnell JM and Korsmeyer SJ: BCL-2 family members and the mitochondria in apoptosis. Genes Dev 13: 1899-1911, 1999.

29. Xie SQ, Wu YL, Cheng PF, et al: A novel homospermidine conjugate inhibits growth and induces apoptosis in human hepatoma cells. Acta Pharmacol Sin 28: 1827-1834, 2007.
30. Xie SQ, Wang JH, Ma HX, Cheng PF, Zhao J and Wang CJ: Polyamine transporter recognization and antitumor effects of anthracenymethyl homospermidine. Toxicology 263: 127-133, 2009.

31. Madeo F, Frohlich E, Ligr M, et al: Oxygen stress: a regulator of apoptosis in yeast. J Cell Biol 145: 757-767, 1999.

32. Voehringer DW: BCL-2 and glutathione: alterations in cellular redox state that regulate apoptosis sensitivity. Free Radic Biol Med 27: 945-950, 1999.

33. Armstrong JS, Steinauer KK, Hornung B, et al: Role of glutathione depletion and reactive oxygen species generation in apoptotic signaling in a human B lymphoma cell line. Cell Death Differ 9: 252-263, 2002.

34. Casero RA Jr and Marton LJ: Targeting polyamine metabolism and function in cancer and other hyperproliferative diseases. Nat Rev Drug Discov 6: 373-390, 2007.

35. Miyajima A, Nakashima J, Yoshioka K, Tachibana M, Tazaki H and Murai M: Role of reactive oxygen species in cis-dichlorodiammineplatinum-induced cytotoxicity on bladder cancer cells. Br J Cancer 76: 206-210, 1997.

36. Zafarullah M, Li WQ, Sylvester J and Ahmad M: Molecular mechanisms of $\mathrm{N}$-acetylcysteine actions. Cell Mol Life Sci 60: 6-20, 2003.

37. Bolkenius FN and Seiler N: Acetylderivatives as intermediates in polyamine catabolism. Int J Biochem 13: 287-292, 1981.

38. Ha HC, Woster PM, Yager JD and Casero RA Jr: The role of polyamine catabolism in polyamine analogue-induced programmed cell death. Proc Natl Acad Sci USA 94: 11557-11562, 1997.

39. Maccarrone M, Bari M, Battista N, Di Rienzo M, Falciglia K and Finazzi Agro A: Oxidation products of polyamines induce mitochondrial uncoupling and cytochrome $\mathrm{c}$ release. FEBS Lett 507: 30-34, 2001

40. Wang Y, Hacker A, Murray-Stewart T, et al: Properties of recombinant human N1-acetylpolyamine oxidase (hPAO): potential role in determining drug sensitivity. Cancer Chemother Pharmacol 56: 83-90, 2005.

41. Lawson KR, Marek S, Linehan JA, et al: Detoxification of the polyamine analogue $\mathrm{N}^{1}$-ethyl- $\mathrm{N}^{11}$-[(cycloheptyl)methy]-4,8diazaundecane (CHENSpm) by polyamine oxidase. Clin Cancer Res 8: 1241-1247, 2002. 